Revista Perspectiva Online: Humanas \& Sociais Aplicadas Julho de 2017, Vol.7, no 19, p.39-47

ISSN: 2236-8876 (Online)

\title{
A ABORDAGEM DE ESTUDO DE CASO E A ÁREA DE ARQUITETURA, URBANISMO E DESIGN: CONSIDERAÇÕES A PARTIR DA ANÁLISE DE UM PERIÓDICO QUALIS A2
}

\author{
Alber Neto $^{1^{*}}$ \& Iago Bersot Arruda ${ }^{2}$
}

\begin{abstract}
RESUMO
NETO, A.S., ARRUDA, I. B. A Abordagem de Estudo de Caso e a Área de Arquitetura, Urbanismo e Design: Considerações a partir da Análise de um Periódico Qualis A2. Perspectivas Online: Exatas \& Engenharias, v. 7 , n.10, p.39-47,2017.
\end{abstract}

Este artigo trata do uso recorrente da abordagem de estudo de caso em produções acadêmicas / científicas em Cursos de Arquitetura e Urbanismo e Cursos de Design. Para tal, o objetivo traçado está em analisar de forma qualiquantitativa a produção publicada entre os anos de 2011 a 2016 em um mesmo periódico nacional Qualis CAPES
A2 em Arquitetura, Urbanismo e Design. Em 306 artigos analisados, foram identificados 77 artigos baseados em estudo de caso $(25,16 \%)$. Assim, aborda-se as características mais recorrentes presentes nestes 77 artigos em um paralelo com um constructo conceitual acerca da abordagem de estudo de caso.

Palavras-chave: Arquitetura; Urbanismo; Design; Estudo de Caso. 


\begin{abstract}
This article addresses the recurrent use of the case study approach in academic / scientific productions in Architecture and Urbanism Courses and Design Courses. To this end, the objective is to qualitatively and quantitatively analyze the production published between the years 2011 and 2016 in a periodical Qualis

CAPES A2 in Architecture, Urbanism and Design. In 306 articles analyzed, 77 articles were identified based on a case study $(25.16 \%)$. The present article addresses the characteristics identified in these 77 articles in a parallel with a conceptual construct formatted from the definitions about case study of different authors.
\end{abstract}

Keywords: Architecture; Urbanism; Design; Case Study.

${ }^{1}$ Institutos Superiores de Ensino do CENSA - ISECENSA - Laboratório de Estudos do Objeto- Rua Salvador Correa, 139, Centro, Campos dos Goytacazes, RJ, CEP: 28035-310, Brasil;

${ }^{2}$ Acadêmico de Arquitetura e Urbanismo ISECENSA- Institutos Superiores de Ensino do CENSA - ISECENSA - Rua Salvador Correa, 139, Centro, Campos dos Goytacazes, RJ, CEP: 28035-310, Brasil.

(*)e-mail: alberneto@gmail.com

Data de chegada: 19/04/2017 Aceito para publicação: 09/06/2017

Persp. online: hum. \& sociais aplicada., Campos dos Goytacazes, 19 (7), 39-47, 2017

seer.perspectivasonline.com.br 


\section{INTRODUÇÃO}

Desde o ano de 2009 a Área de Arquitetura e Urbanismo na Coordenação de Aperfeiçoamento de Pessoal de Nível Superior (CAPES) passou a ser intitulada de Arquitetura, Urbanismo e Design. Para além dos vínculos históricos entre (i) Arquitetura e Urbanismo e (ii) Design, estas áreas se intersecionam e possuem epistemologia comum, permitindo tal classificação para a produção intelectual (CAPES, 2009; DROSTE, 2013).

Ambas diretrizes curriculares de Arquitetura e Urbanismo e de Design versam sobre os aspectos tecnicistas: conhecimento de leis e normas, raciocínio lógico e matemático, geometria, domínio de softwares, ergonomia; versam de suas correlações com as engenharias: entendimento das técnicas construtivas e produtivas. E, deve-se destacar que, ambas dão ênfase aos aspectos humanísticos: foco no usuário (comportamento e cultura), métodos criativos, história e habilidades artísticas a fim de propiciar fruição estética (MEC, 2004; MEC, 2010).

Quando da prática projetual, os profissionais destas áreas — arquitetos e designers — iniciam com a definição de um problema. Este problema é composto de variáveis técnicas e funcionais (aspectos tecnicistas), e variáveis abstratas (aspectos humanísticos). Estas últimas se referem ao partido arquitetônico e traços culturais, por exemplo, e que condicionam as características morfológicas finais de um determinado produto e que influenciam - por sua vez — na usabilidade, funcionamento aceitação por parte do público, etc.

Pode-se dizer que a palavra problema, refere-se a um conjunto de necessidades que devem ser atendidas ao fim de um projeto. Logo, um desenho técnico de uma casa pode ser a solução de um problema em Arquitetura, assim como o desenho técnico de cadeira pode ser a solução de um problema de um projeto de Design.

Já os componentes de um problema de projeto são as variáveis que referem as necessidades específicas que devem ser resolvidas na solução final que marcará o encerramento do projeto. Retomando o exemplo da casa, considerando o componente do problema que se refere ao público a ser atendido, deve-se observar que um projeto que visa acomodar uma família - composta por um casal e dois filhos - pertencente a uma alta classe econômica, tende a ser diferente de um outro projeto que acomodará uma família com a mesma configuração pertencente a uma faixa econômica de baixa renda. Portanto, a identificação e delimitação do problema e de seus componentes (requisitos funcionais específicos, leis e normas pertinentes, características culturais, predileções pessoais e etc.) é fundamental para iniciar o desenvolvimento de uma solução adequada para um caso específico.

Dada a particularidade de cada caso em Arquitetura, Urbanismo e Design, e de cada resultado de projeto proposto para solucionar cada problema específico, afirma-se que "estudo de caso é um dos métodos mais comuns na área" (SERRA, 2006, p. 82).

A fim de ilustrar a compreensão apresentada até o momento, cita-se o caso da habitação de interesse social de autoria de Elemental. O modelo de casa foi projetado a partir da doutrina de Design Participativo, ou seja, quando o projeto é orientado de modo a atender as especificidades do público alvo em questão, sendo este os cidadãos da comunidade de Lo Barnechea (em Santiago, Chile). A casa entregue às famílias é incompleta, no sentido de que prever alterações e expansões de acordo com as necessidades de cada núcleo familiar. Na primeira versão entregue às famílias, é interessante observar que o banheiro poderia ter uma banheira ou um aquecedor de água. Os projetistas e assistentes sociais envolvidos estimavam que a respostas em sua totalidade apontariam para o aquecedor, visto as baixas temperaturas da região. Mas, ao interagir com o público, foi exatamente o contrário. Uma vez que população atendida vivia em condições insalubres e se acostumará com as baixas temperaturas quando de sua higiene pessoal, preferiam a banheira. Outro ponto que fora detectado contra o aquecedor foi o inevitável aumento do gasto de energia que o mesmo representaria - e que as famílias não poderiam arcar. O estudo minucioso do caso, considerando os aspectos humanísticos, foi preponderante para o sucesso do projeto (ARAVENA, 2011).

Persp. online: hum. \& sociais aplicada., Campos dos Goytacazes, 19 (7), 39-47, 2017

seer.perspectivasonline.com.br 
Isto ocorre por que ambas as Áreas, Arquitetura e Urbanismo e Design, integrantes das Ciências Sociais Aplicadas, têm natureza propositiva. Assim, uma vez identificado um problema, o projetista considera os aspectos técnicos e humanísticos a fim de determinar a forma final do projeto a partir daquela doutrina que adota. Ou seja, a quantidade de soluções diferentes e funcionais para um mesmo problema poderá ser diretamente proporcional a quantidade de projetistas que se propuserem a solucioná-lo.

Para além dos aspectos tecnicistas e de sua inegável preponderância, fica evidente o caráter qualitativo destas ciências quando da importância dos aspectos humanísticos das mesmas. E considerando a supracitada natureza propositiva da Área de Arquitetura e Urbanismo e da Área de Design, onde cada caso é necessariamente singular e deve ser entendido de forma específica, pesquisas qualitativas são valiosas no processo de evolução de doutrinas. Ou seja, diferente de outras ciências que buscam formatar e operar teorias gerais, nas Ciências Sociais Aplicadas é comum lançar mão de pesquisas de caráter qualitativo para estudo e entendimento profundo de determinados casos e seus desdobramentos (SERRA, 2006; IDEO, 2013).

Dado a supracitada relevância que a abordagem de estudo de caso possui para a Arquitetura, Urbanismo e Design, este artigo tem como objetivo formatar um constructo teórico acerca da mesma e realizar uma analise quantitativa dos artigos publicados em um periódico Qualis CAPES A2 a fim de verificar a incidência de produções que se valem desta abordagem e as principais características que as definem.

\section{METODOLOGIA}

Em um primeiro momento, caracteriza-se o objetivo da pesquisa relatada neste artigo como exploratória, uma vez que não visa esgotar o problema abordado - ou seja, não visa apresentar uma único conjunto de características que um estudo de caso deva ter. Os dados utilizados partem de uma documentação indireta, seja no levantamento de conceitos e definições, seja quando da formação de um banco de dados correspondente aos artigos publicados em um periódico Qualis CAPES A2. O tratamento dos dados se dá por uma abordagem quali-quantitativa.

Para além dos conceitos e definições levantados em Bennett (2015), Ganga (2012), Orum (2015), Serra (2006) e Yin (2015), destaca-se as produções de Hyett, Kenny e Dickson-Swift (2014) e Runfola et al. (2016) que também se propõem a analisar o quantitativo de artigos sustentados por estudos de caso em periódicos de suas áreas.

Sobre a escolha do periódico, afirma-se que a mesma se deu pela sua linha editorial que de fato abrange a totalidade da área de Arquitetura, Urbanismo e Design (AUD). Indo de encontro com a fragilidade apontada pela CAPES (2015), "Discreta participação de periódicos nacionais nos estratos A1 e A2", $\square$ menciona-se a inexistência de periódicos nacionais no estrato A1 e a maior parte de periódicos A1 e A2 sem adesão real á área de AUD, como - por exemplo - sugerem os seguintes títulos: Child's Nervous System (A1), Reproduction In Domestic Animals (A1), Arquivo Brasileiro de Medicina Veterinária e Zootecnia (A2), e Revista Brasileira de Reumatologia (A2).

Assim, analisou-se a produção veiculada em tal periódico — até o momento - na presente década de 2010, sendo 306 artigos entre 2011 e 2016. Além de identificar os artigos baseados em estudo de caso, destes buscou-se analisar o número de exemplares do objeto de estudo que fora considerado, onde tal abordagem fora citada, o tipo de documentação (direta ou indireta), e o tipo de recursos utilizado para relatar o estudo de caso proposto.

Persp. online: hum. \& sociais aplicada., Campos dos Goytacazes, 19 (7), 39-47, 2017

seer.perspectivasonline.com.br 


\section{DISCUSSÃO}

Baseando-se nos autores Bennett (2015), Ganga (2012), Hyett, Kenny e Dickson-Swift (2014), Orum (2015), Runfola et al. (2016), Serra (2006) e Yin (2015), considerando a interseção acerca daquilo que é versado sobre estudo de caso, pode-se dizer que:

- É correto e seguro afirmar que não existe uma única definição sobre o que seja estudo de caso. Existe um consenso, no entanto, que um estudo de caso busca extrair o máximo de um determinado objeto de estudo a partir de uma determina doutrina (sendo este objeto algo tácito ou não, um produto ou um fenômeno). Portanto, um mesmo objeto de estudo pode ser utilizado em estudos de casos distintos que, por sua vez, consideram doutrinas distintas;

- Se um estudo de caso tende a apresentar conclusões pontuais, uma coleção de estudos de caso a partir de uma determinada doutrina podem vir a sustentar a formulação de uma teoria;

- Trata-se de um abordagem qualitativa. E para tal, a análise do objeto de estudo deve ser pautada — invariavelmente — por critérios previamente definidos e que estejam compreendidos pela doutrina a ser utilizada;

- Estudos de caso são denominados de estudos de baixo $n$, ou seja, considera-se um exemplar de um objeto de estudo ou poucos exemplares que atendam a caracterização dada ao objeto de estudo. Um único exemplar de um estudo de caso pode conter partes quantificáveis, por exemplo, podendo o pesquisador lançar mão de uma análise qualiquantitativa;

- O pesquisador deve considerar o ambiente onde o objeto de estudo está inserido e buscar estar presencialmente junto ao mesmo. Todavia, realizar documentação direta não se trata de uma regra.

A partir desta última afirmação, cita-se - especificamente - Hyett, Kenny e Dickson-Swift (2014) que discutem o estudo de caso enquanto abordagem de pesquisa e não como metodologia. Sendo o estudo de caso adotado pelas mais diversas áreas, a forma como o estudo é planejado (etapas e ferramentas) tende a variar demasiadamente. Entretanto, esta mesma liberdade metodológica acaba por fragilizar parte das pesquisas. Citam que parte considerável dos artigos baseados em estudo de caso não delimitam de forma satisfatória o objeto de estudo, não especificam os critérios a serem considerados quando da análise do objeto de estudo, e não descrevem de forma coerente as etapas e ferramentas de pesquisa (ou seja, a metodologia). E este tipo de inconsistência praticada tende a gerar uma percepção negativa acerca dos artigos que se valem de estudo de caso, o que também é versado por Runfola et al. (2016).

Para além do supracitado constructo conceitual, como dito anteriormente, entre os anos de 2011 e 2016 foram publicados 306 artigos no periódico analisado, este classificado como Qualis CAPES A2. Nesta amostragem de 306 artigos, encontrou-se 77 artigos $(25,16 \%)$ que se valeram da abordagem de estudo de caso (figura 1).

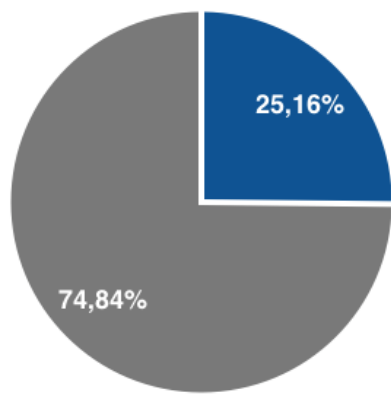

Estudo de caso (77 de 306)

Outras abordagens (229 de 306)

FIGURA 1. Proporção de artigos baseados em estudo de caso. Fonte: figura nossa (2017).

Persp. online: hum. \& sociais aplicada., Campos dos Goytacazes, 19 (7), 39-47, 2017

seer.perspectivasonline.com.br 
Considerando então este universo de 77 artigos, ao analisar a estruturação dos mesmos, pode-se perceber que o estudo de caso é mencionado com maior incidência no item de método (76,62\%), seguido de resumo (42,86\%), introdução (37,66\%), referencial teórico $(29,87 \%)$, e título $(22,08 \%)$ - vide figura 2.

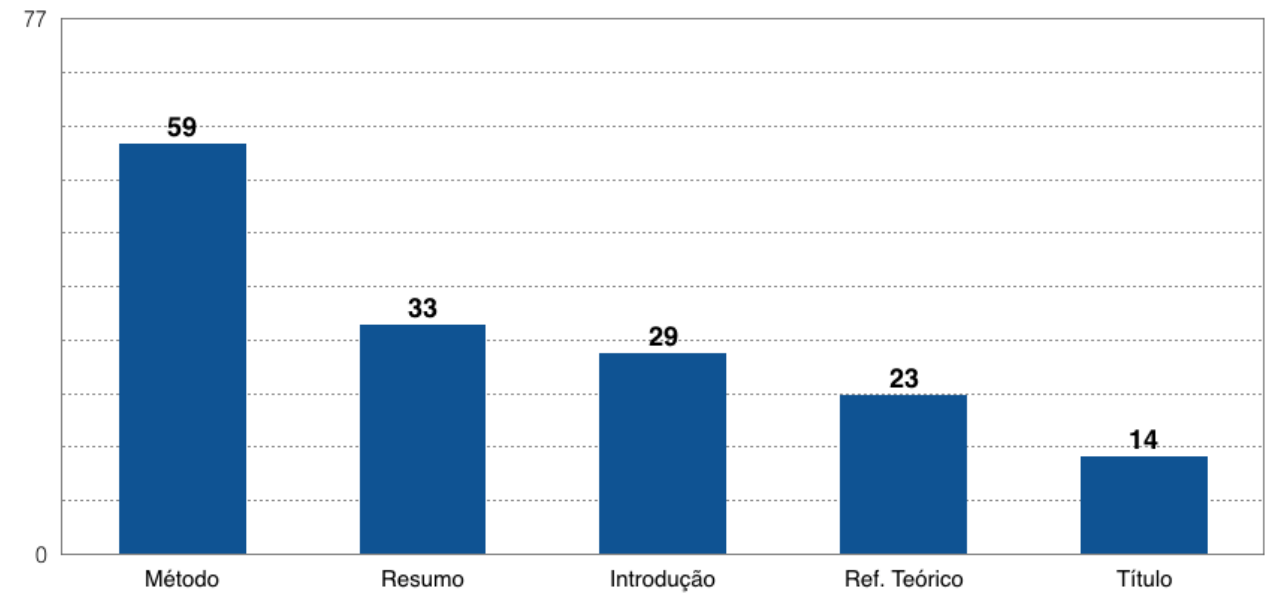

FIGURA 2. Partes do artigo onde o estudo de caso fora mencionado com maior incidência $(n=77)$. Fonte: figura nossa (2017).

Por sua vez, o objeto do estudo fora - em sua maioria - composto por um único exemplar (51,95\%), o que remete a abordagens descritivas e/ou explicativas. Em seguida, percebe-se que os estudos com dois ou três exemplares, que remetem a abordagens também comparativas, possuem uma segunda fatia significativa com $29,87 \%$. Os artigos que consideram 4 ou mais exemplares correspondem a $18,18 \%$ dentre os 77 artigos analisados (figura 3).

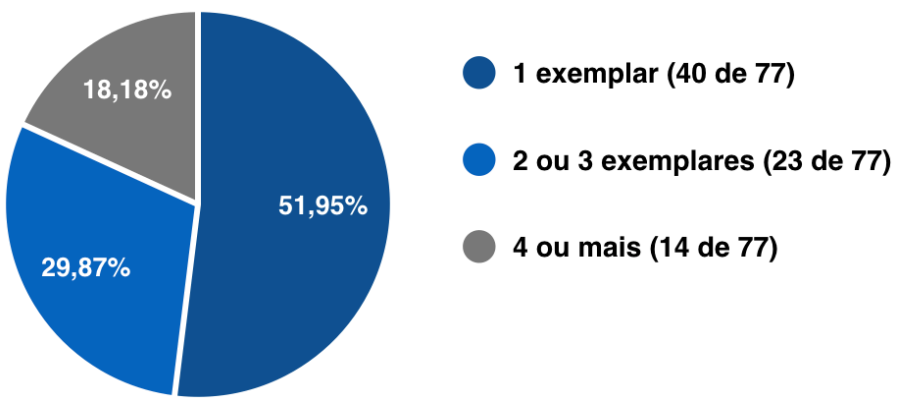

FIGURA 3. Número de exemplares do objeto do estudo de caso ( $n=77)$. Fonte: figura nossa (2017).

Deve-se dizer que a maioria dos artigos baseados em estudo de caso se valeram de uma documentação direta de seus respectivos objetos de estudo. Foram 57 artigos dos 77 analisados (74,03\%), frente a 20 de $77(25,97 \%)$ que se valeram de documentação indireta (figura 4).

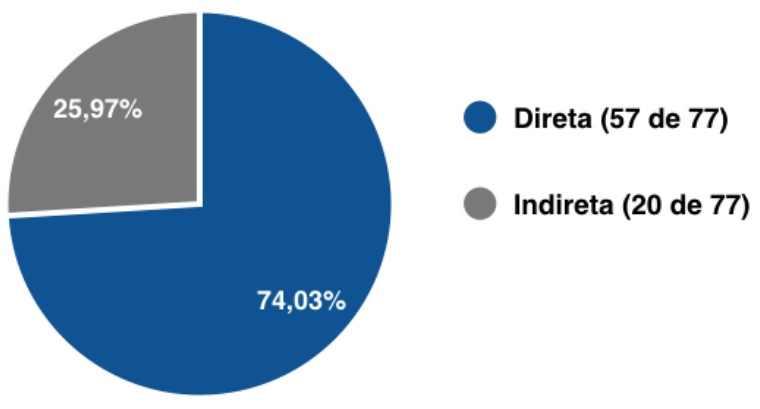

FIGURA 4. Número de exemplares do objeto do estudo de caso $(n=77)$. Fonte: figura nossa (2017).

Persp. online: hum. \& sociais aplicada., Campos dos Goytacazes, 19 (7), 39-47, 2017 
Por fim, afirma-se que os artigos baseados em estudos de caso utilizam recursos gráficos em demasia (figura 5). Tabelas têm maior incidência $(90,91 \%)$ e são seguidas por esquemas gráficos $(84,42 \%)$, gráficos $(59,74 \%)$, e fotos $(58,44 \%)$. Entende-se como esquemas gráficos, por exemplo, diagramas, fluxogramas e plantas de prédios.

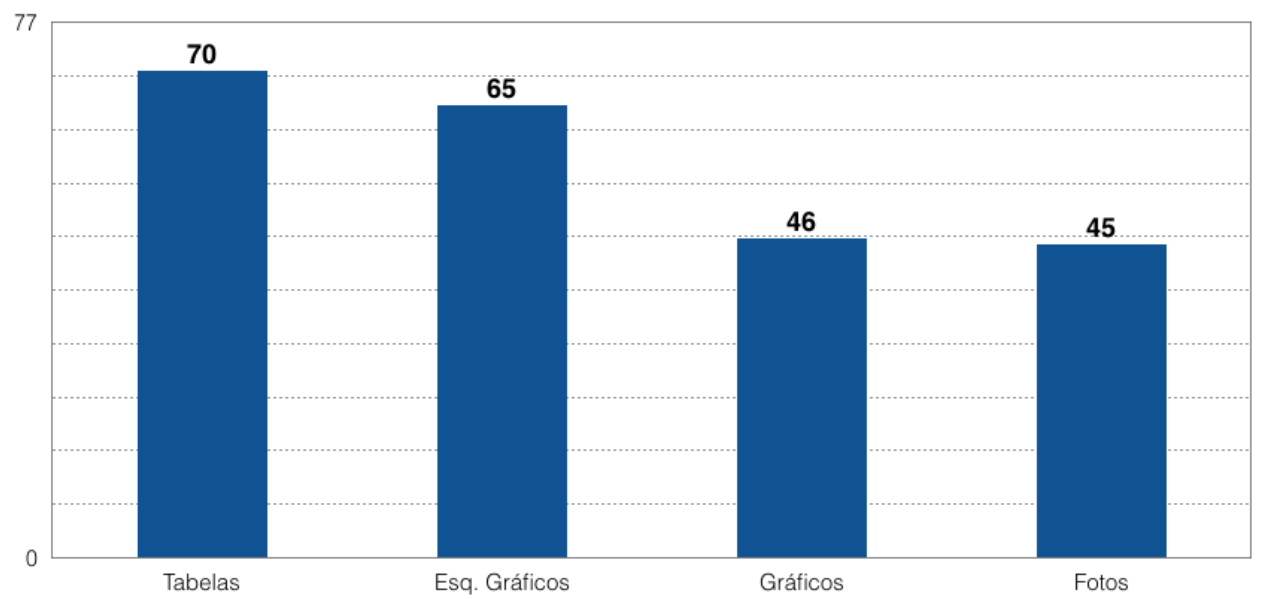

FIGURA 5. Incidência da utilização de recursos gráficos ( $n=77)$. Fonte: figura nossa (2017).

A seguir, apresenta-se uma interpretação desta analise a partir do constructo conceitual formatado.

\section{CONSIDERAÇÕES FINAIS}

A partir de diversos autores foi possível formatar um constructo conceitual coerente acerca da abordagem de estudo de caso. Assim, considerando tal constructo, as críticas encontradas em Hyett, Kenny e Dickson-Swift (2014) e Runfola et al. (2016) - que conduziram estudos similares ao proposto neste artigo, e a análise dos 77 (de 306) artigos que se valeram de estudos de caso e foram publicados entre os anos de 2011 a 2016 no periódico Qualis CAPES A2, é possível dizer que:

- Estudo de caso é uma abordagem usada com recorrência na área de Arquitetura, Urbanismo e Design (25,16\%), como supracitado por meio de Serra (2006);

- A abordagem de estudo de caso é mencionada com mais frequência no item que se refere ao método (76,62\%). Para além de citar conceitos e definições sobre a abordagem de estudo de caso, deve-se delimitar com precisão o objeto de estudo, especificar os critérios a serem considerados quando da análise deste objeto, e descrever de forma coerente as etapas e ferramentas de pesquisa (ou seja, a metodologia);

- Já o título é o item com menor incidência $(22,08 \%)$ de menção à abordagem de estudo de caso. Uma vez que um texto científico deve ser sóbrio e pragmático, pensa-se que o título possa revelar o tipo de abordagem utilizada naquele artigo;

- Estudos de caso são denominados de estudos de baixo $n$ e buscam extrair o máximo de um determinado objeto de estudo a partir de uma determina doutrina. Neste sentido, percebe-se que a maior parte dos artigos abordam um único objeto de estudo $(51,95 \%)$. Os artigos que consideram dois ou três exemplares que atendem a delimitação do objeto de estudo também são recorrentes $(29,87 \%)$. Os artigos que se valem de quatro ou mais exemplares correspondem a uma menor parcela $(18,18 \%)$ - isto pois tendem a serem superficiais quando da análise desses diversos exemplares;

- A realização de documentação direta não é uma regra. Entretanto, identifica-se que, na maior parte dos artigos $(74,03 \%)$, o pesquisador buscou a documentação direta de informações sobre $p$ objeto de estudo;

Persp. online: hum. \& sociais aplicada., Campos dos Goytacazes, 19 (7), 39-47, 2017 
- Recursos gráficos são muito quando de estudos de caso.

O quantitativo expressivo de artigos que se valem da abordagem de estudo de caso corrobora o mote aqui levantado. A proposta deste artigo não está em delimitar as características de um estudo de caso quando da produção científica na área de Arquitetura, Urbanismo e Design. Entretanto, deve-se notar que as características identificadas nos 77 artigos analisados dialogam com o constructo conceitual formatado. A abordagem de estudo de caso permite diferentes formatos de metodologia, e essa mesma liberdade por vezes interfere na qualidade dos artigos. As características identificadas, então, podem ser uma orientação principalmente para jovens pesquisadores — mas não um padrão.

Especificamente, nos 77 artigos analisados, percebe-se um maior número de estudos de caso que se valem de documentação direta. Existe a possibilidade de realizar um estudo de caso por meio de documentação indireta, mas sempre se atentando a quantidade e qualidade de material / informação disponibilizado que pode ser utilizado para o estudo proposto. Pensa-se que a publicação de estudos de caso sem esmero metodológico, mesmo em periódicos de menor impacto, tendem a banalizar a abordagem.

Visto a necessidade de intensificar as práticas e processos de investigação científica na Área de Arquitetura, Urbanismo e Design apontadas por Amorim (2012), Rocha-Peixoto e Cabral (2012) e CAPES (2013), considerando a importância da técnica de estudo de caso para a Área, a pesquisa apresentada neste artigo avançara para a análise de outros periódicos indexados ao Qualis CAPES a fim de apresentar outras recomendações e debater o estudo de caso enquanto abordagem e suas implicações metodológicas.

\section{REFERÊNCIAS}

AMORIM, Luiz. A pesquisa na área de arquitetura e urbanismo: um quadro a partir do CNPq. In: ROCHAPEIXOTO, Gustavo; CABRAL, Maria Cristina; AZEVEDO, Marlice. Difusão de pesquisa em Arquitetura e Urbanismos. Rio de Janeiro: Rio Books / CAPES, 2012.

ARAVENA, Alejandro. About Social Housing In Lo Barnechea. In: Gary Hustwit. Urnbanized. 2011.

BENNETT, Andrew. Case Study: Methods and Analysis. International Encyclopedia of the Social \& Behavioral Sciences. v.3, n.2, p. 208-213, 2015. Disponível em: <sciencedirect.com>. Acesso em: 10 mar. 2017.

CAPES. Considerações Gerais sobre o atual estágio da Área de Arquitetura, Urbanismo e Design. Coordenação de Aperfeiçoamento de Pessoal de Nível Superior (CAPES). 2009. Disponível em:

<www.capes.gov.br>. Acesso em: 23 maio 2016.

Considerações Gerais sobre o atual estágio da Área de Arquitetura, Urbanismo e Design. Coordenação de Aperfeiçoamento de Pessoal de Nível Superior (CAPES). 2013. Disponível em: <www.capes.gov.br>. Acesso em: 23 maio 2016.

Seminário Síntese Acompanhamento de Meio Termo SNPG. Coordenação de Aperfeiçoamento de Pessoal de Nível Superior (CAPES). 2015. Disponível em: <www.capes.gov.br>. Acesso em: 10 mar. 2017.

DROSTE, Magdalena. Bauhaus: Bauhaus Archiv. Tradução: Casa das Línguas. Taschen: 2013.

GANGA, Gilberto Miller Devós. Trabalho de Conclusão de Curso (TCC) na engenharia de produção: um guia prático de forma e conteúdo. São Paulo: Atlas, 2012.

MEC. Resolução $\mathrm{n}^{\circ}$ 5, de 8 de março de 2004. Aprova as Diretrizes Curriculares Nacionais do Curso de Graduação em Design e dá outras providências. Diário Oficial da República Federativa do Brasil. Brasília, DF. Disponível em: <http://www.mec.gov.br>. Acesso em: 23 maio 2016.

Persp. online: hum. \& sociais aplicada., Campos dos Goytacazes, 19 (7), 39-47, 2017

seer.perspectivasonline.com.br 
Resolução no 2, de 17 de junho de 2010. Institui as Diretrizes Curriculares Nacionais do curso de graduação em Arquitetura e Urbanismo, alterando dispositivos da Resolução CNE/CES no 6/2006. Diário Oficial da República Federativa do Brasil. Brasília, DF. Disponível em: <http://www.mec.gov.br>. Acesso em: 23 maio 2016.

HYETT, Nerida; KENNY, Amanda; DICKSON-SWIFT, Virginia. Methodology or method? A critical review of qualitative case study reports. International Journal of Qualitative Studies on Health and Well-being. v.9, n.1, p. 1-12, 2014. Disponível em: <sciencedirect.com>. Acesso em: 10 mar. 2017.

IDEO. Human Centered Design: kit de ferramentas. 2 ed. Título original: Human Centered Design: toolkit. Tradução: Tennyson Pinheiro, José Colucci Jr., Isabela de Melo. IDEO: 2013. Disponível em: $<$ www.IDEO.com>. Acesso em: 23 maio 2016.

ORUM, Anthony M . Anthony M. Case Study: Logic. International Encyclopedia of the Social \& Behavioral Sciences. v.3, n.2, p. 202-207, 2015. Disponível em: <sciencedirect.com>. Acesso em: 10 mar. 2017.

ROCHA-PEIXOTO, Gustavo; CABRAL, Maria Cristina. Arquitetura como árvore: à guise de introdução. In: ROCHA-PEIXOTO, Gustavo; CABRAL, Maria Cristina; AZEVEDO, Marlice. Difusão de pesquisa em Arquitetura e Urbanismos. Rio de Janeiro: Rio Books / CAPES, 2012.

RUNFOLA, Andrea et al. The use of qualitative case studies in top business and management journals: a quantitative analysis of recent patterns. European Management Journal. v.35, n.1, p. 116-127, 2016. Disponível em: <sciencedirect.com>. Acesso em: 10 mar. 2017.

SERRA, Geraldo G. Pesquisa em Arquitetura e Urbanismo: guia prático para o trabalho de pesquisadores em pós-graduação. São Paulo: Edusp, 2006.

YIN, Robert K. Case Studies. International Encyclopedia of the Social \& Behavioral Sciences. v.3, n.2, p. 194-201, 2015. Disponível em: <sciencedirect.com>. Acesso em: 10 mar. 2017.

Persp. online: hum. \& sociais aplicada., Campos dos Goytacazes, 19 (7), 39-47, 2017 